\title{
CREEP OF GEOMATERIALS DUE TO COUPLED DAMAGE AND SPONTANEOUS MINERAL DISSOLUTION
}

\author{
Liang Bo Hu${ }^{1}$ and Tomasz Hueckel ${ }^{1}$ \\ ${ }^{1}$ Duke University, Department of Civil and Environmental Engineering \\ Durham, NC 27708-0287, USA \\ e-mails: 1h19@duke.edu; hueckel@eduke.edu
}

Keywords: creep, damage, chemo-plasticity, mineral dissolution

\begin{abstract}
A basic model of chemical softening, and chemically enhanced deviatoric strain hardening for saturated geomaterials is presented. The aim is to simulate the material behavior that exhibits characteristics of creep induced by environmental conditions. Chemical softening is postulated to occur as a consequence of mineral dissolution and precipitation enhanced by the material damage. Dissolution of minerals in pore water is assumed to take place at the internal free surfaces not only of the initial pore space but also that generated during damage. The rate dependence of the processes of dissolution and precipitation renders also the resulting chemical softening behavior to be rate dependent. In this paper closed system is discussed only, in which deformation at constant stress results entirely a from a local compensation mechanism between the chemical softening and strain hardening. Three stages of creep are interpreted in terms of mechanisms of dissolution and precipitation, as well as the variation in the reaction area involved in the mass exchange. An open system, these local mechanisms are enhanced by diffusion of species affecting the mass balance and need to be addressed via a boundary value problem, as described elsewhere [31].
\end{abstract}




\section{INTRODUCTION}

Creep is known to be a process of continuous straining exhibited by a material at macroscopic scale at constant stress. A series of phenomena identifiable at the micro-scale have been indicated as possible sources of such macroscopic manifestation. For metals, these include dislocation glide or climbing, Nabarro-Herring creep resulting from intra-grain diffusion and Coble creep produced by diffusion along the grain boundaries [1]. For soils, early efforts in this direction were undertaken by Murayama and Shibata [2], Christensen and Wu [3], Mitchell [4] Kuhn and Mitchell [5].

In this contribution a hypothesis is presented that one of the mechanisms of creep in geomaterials arises due to a chemically sustained mechanical damage process. The motivation for that hypothesis stems from the observation that the macroscopic creep occurs after some amount of damage is caused in the material. A slow pace of the deformation initiated by that damage is commensurate with a simultaneous, rate driven chemical process. In the considered case, a time-dependent chemical enhancement occurs through dissolution of minerals from a zone of dilatant damage. This damage is known to be associated with micro-cracking (see e.g. Brace et al. [6]). Micro-cracking generates a new surface area at the crack walls. In contact with an interstitial fluid, these walls become new dissolution sites. The removal of the mineral mass via dissolution leads to an ulterior material softening and further deformation at constant stress.

The presented mechanism has been inspired by one of the hypotheses of the pressure solution in compacting rocks, such as halite and sandstone. Experiments and field evidence corroborating this hypothesis were developed among others by Gratz [7], Tada and Siever [8], and Milliken [9]. Microcracks near the intergranular contact are suggested (den Brok [10]) to be the precursors of the well-known structure of channels and islands etched in the contact surfaces (see e.g. Schutjens and Spiers [11]). Fig. 1 (from He et al. [12]) shows an SEM image of the dissolution sites at grain contacts stressed to $34.5 \mathrm{MPa}$ at $150^{\circ} \mathrm{C}$ for 14 months. The chemistry of the aqueous environment on the stress enhanced chemical reaction at the crack tip has been intensely investigated in mid ' 80 s as a possible cause of crack propagation at constant load in silicate rocks (see e.g. Atkinson [13]).

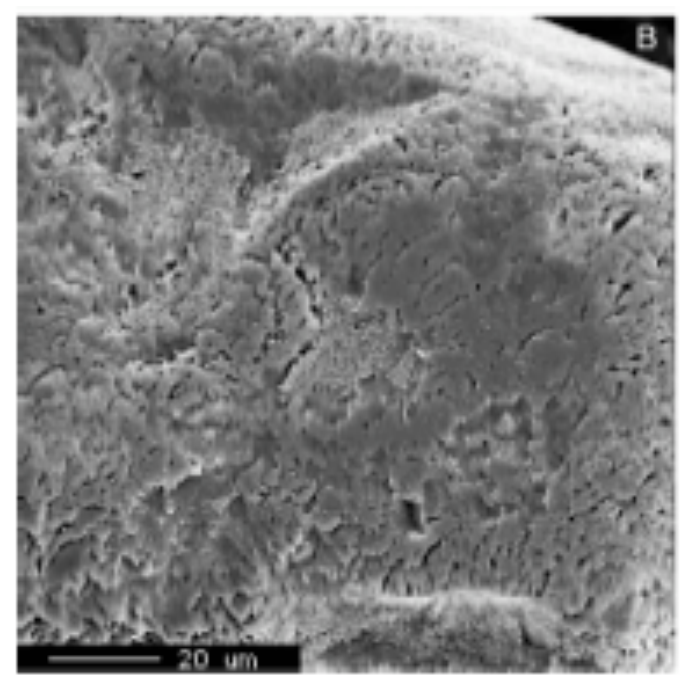

Fig. 1. Dissolution sites at quartz grain contacts stressed to $34.5 \mathrm{MPa}$ at $150{ }^{\circ} \mathrm{C}$ for 14 months: SEM image (from $\mathrm{He}$ et al. [12])

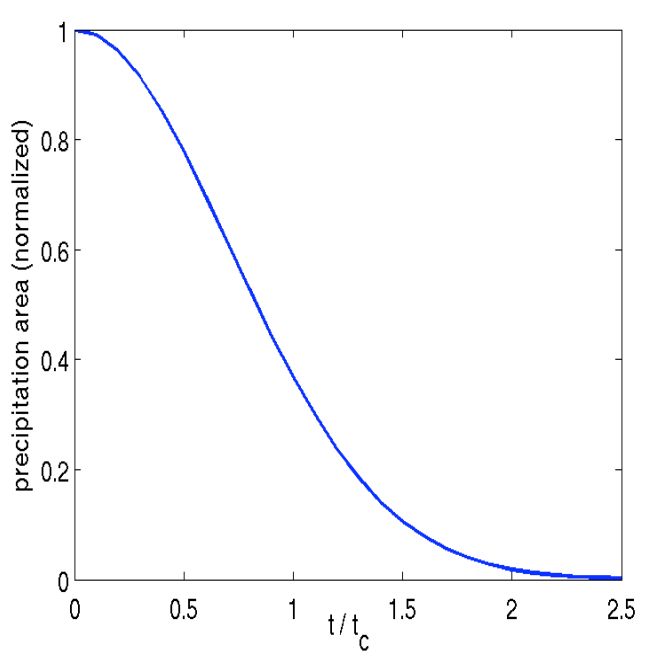

Fig. 2. Normalized precipitation surface area $\tilde{a}_{p} / \tilde{a}_{p 0}^{\prime}$ 


\section{GENERAL CONCEPTS OF COUPLED CHEMO-PLASTICITY}

We shall build on the previously developed idea (Hueckel $[13,14])$ that a mass removed from/added to a solid isochorically (without the volume change of the representative elementary volume of the porous medium) and adiabatically (without heat exchange) affects both the medium compliance and strength. These two effects will be addressed in the framework of what is called reactive chemo-plasticity. The term reactive chemoplasticity Hueckel [15], is used as opposed to non-reactive chemoplasticity (Hueckel [16]) when instantaneous change in concentration (of selected species) of pore fluid may produce change in mechanical properties of the solid phase. This is distinguished in analogy to reactive and non-reactive transport, and is important because of a difference in time scales.

The main assumptions are as follows. The material under consideration is a two-phase multi-component porous solid saturated with water. However, the processes involved are sufficiently slow, so that the material may be considered as perfectly drained. As a consequence, the fluid phase carries no partial stress.

A Representative Elementary Volume (REV) of the porous, water-saturated medium is considered, with the two phases superimposed, and represented by single geometrical point, to which all the mechanical and chemical properties are attributed. To maintain the focus on damage we assume at this stage that the solid phase material is rigid-plastic, with the understanding that a sufficient load is applied to induce initial yielding. This is to say that there is no strain $\left(\varepsilon_{i j}\right)$ for a stress $\sigma_{i j}$ within the yield locus, $f=0$, whereas

at yielding, the strain rate is entirely irreversible

$$
f<0, \varepsilon_{i j}=0 ; f\left[\sigma_{i j}, p_{c}\left(\kappa, \xi_{k}\right)\right]=0 \text { and } \quad \dot{f}\left(\dot{\sigma}_{i j}, \dot{p}_{c}\right)=0 ; \quad \dot{\varepsilon}_{k l}=\dot{\varepsilon}_{k l}^{i r r} \neq 0
$$

where superimposed dot over a symbol denotes its time rate. Stress and strain are considered as positive when in compressive domain. Strains are assumed to be small. $p_{c}$ is a geometric characteristics of the yield locus and is a hardening function which depends on a set of hardening parameters, that are either mechanical $(\kappa)$ or chemical $(\xi)$ in nature (Hueckel [15]). The deviatoric strain hardening parameter $\kappa$ is determined by

$$
\dot{\kappa}=\left(\frac{2}{3} \dot{e}_{i j}^{i r r} \dot{e}_{i j}^{i r r}\right)^{1 / 2}, \text { where } \dot{e}_{i j}^{i r r}=\dot{\varepsilon}_{i j}^{i r r}-\frac{1}{3} \dot{\varepsilon}_{k k}^{i r r} \delta_{i j}
$$

During yielding dilatancy-damage occurs, linked to micro-cracking. Micro-cracking produces new solid-fluid interfaces, which constitute source of dissolution of mineral species resulting in the solid mass removal. As a result the yielding behavior of the material is affected by two plastic hardening mechanisms: deviatoric strain-hardening and mass removal softening.

The set of hardening parameters $\xi_{k}, k=1,23 \ldots$ represents an accumulated relative mass removal/addition of a particular mineral species, $k$, that is deemed to contribute to the material strength or yield limit. The removal is calculated with respect to the original total mass of that particular species. The rates of removal are calculated from the rates of reactions in which the given spoecies is involved. In principle, more than one reaction or chemo-physical process (e.g. osmosis), may affect the strength of any geomaterial. These processes may be simultaneous or sequential, and each with a possibly different rate with which they progress. There is currently no widely accepted approach to the question how to identify and quantify mineral 
component contributions to the overall geomaterial strength or compressibility. Averaging, or homogenization techniques offer a viable tool for that purpose (Murad et al. [17] but are focused on textural inhomogeneity, rather than mineral one. Also, configuration aspects of particular species within the volume may play a critical role, like that of calcium carbonate at the bridges between silica grains. However, in some circumstances, a phenomenological relationship between strength and a reaction progress variable may be established empirically. In what follows we will address a single reaction effect only, with a single rate.

Dissolution is characterized as a kinetic process with the dissolution rate proportional to the specific surface area of the newly generated interfaces. As the latter in turn depends on the dilatancy-damage, the strain hardeninng and chemical softening mechanism are coupled.

As the yield locus for porous material is expected to depend on the mean stress, the associated flow rule according to which the strain rate mode is co-linear with the yield locus gradient is a proper tool to describe strain rate, including irreversible dilatancy, while the amount of the strain rate is controlled by the rate of change of plastic multiplier, $\dot{\lambda}$,

$$
\dot{\varepsilon}_{i j}^{i r r}=\dot{\lambda} \frac{\partial f}{\partial \sigma_{i j}}
$$

Multiplier $\dot{\lambda}$, which depends on the rates of stress, temperature and reaction is determined from the consistency condition, $\dot{f}\left(\sigma_{i j}, \kappa, \xi\right)=0$,

$$
\frac{\partial f}{\partial \sigma_{i j}} \dot{\sigma}_{i j}+\frac{\partial f}{\partial \kappa} \dot{\kappa}+\frac{\partial f}{\partial \xi} \dot{\xi}=0
$$

Hence,

$$
\dot{\lambda}=-\frac{\frac{\partial f}{\partial \sigma_{m n}} \dot{\sigma}_{m n}+\frac{\partial f}{\partial \xi} \dot{\xi}}{\frac{\partial f}{\partial \kappa}\left[\frac{2}{3} \frac{\partial f}{\partial s_{i j}} \frac{\partial f}{\partial s_{i j}}\right]^{1 / 2}}
$$

whereas $s_{i j}=\sigma_{i j}-\frac{1}{3} \sigma_{k k} \delta_{i j}$.

For creep, and hence at $\dot{\sigma}_{i j}=0$, the creep strain rate becomes proportional to the rate of the relative mass removal

$$
\dot{\varepsilon}_{i j}^{i r r}=-\frac{\frac{\partial f}{\partial \sigma_{i j}} \frac{\partial f}{\partial \xi} \dot{\xi}}{\frac{\partial f}{\partial \kappa}\left[\frac{2}{3} \frac{\partial f}{\partial s_{i j}} \frac{\partial f}{\partial s_{i j}}\right]^{1 / 2}}
$$

As may be seen, during creep the two hardening mechanisms in (eq. 4) become compensatory, and hence, their rates are linearly dependent. The chemical dissolution induces further damage enhancement: this results in a two-way coupling. Compensatory mode is common whenever an environmental softening is present, such as one due to temperature (Hueckel and Borsetto [18]), concentration (Hueckel [19]), mass transfer (Hueckel [15]), or suction 
(Liu et al. [19]).

\section{CHEMICALLY INDUCED MASS REMOVAL}

In what follows dissolution of pure silica in contact with water in a geological system will be considered.

$$
\mathrm{SiO}_{2}(s)+2 \mathrm{H}_{2} \mathrm{O}(l) \leftrightarrow \mathrm{H}_{4} \mathrm{SiO}_{4}(a q)
$$

The rate of this reaction is directly proportional to the area of the interface between the two phases. For a system, which contains mass of water $M=M^{0}=1 \mathrm{~kg}$, the fluid/solid interface surface area, $A$, is normalized with respect to $A^{0}=1 \mathrm{~m}^{2}$ to yield a non-dimensional quantity $\tilde{A}$ $=A / A^{0}$. The mass loss of silica from the solid phase is expressed, following Rimstidt and Barnes [21], via activity (mass) of silicic acid, $\mathrm{H}_{4} \mathrm{SiO}_{4}$, dissolved in water. This has been directly measured in an experiment in a closed environment. under the assumption the interfacial area between the phases remained the same in the case of dissolution and precipitation, yielding a rate equation for silica interaction with water

$$
\frac{d a_{\mathrm{H}_{4} \mathrm{SiO}_{4}}}{d t}=\tilde{\mathrm{A}} \gamma_{\mathrm{H}_{4} \mathrm{SiO}_{4}}\left(k_{+} a_{\mathrm{SiO}_{2}} a_{\mathrm{H}_{2} \mathrm{O}}^{2}-k_{-} a_{\mathrm{H}_{4} \mathrm{SiO}_{4}}\right)
$$

where $a_{i}$ are activities, and $\gamma_{i}$, activity coefficients, of $i$-th species, while $k_{+}$and $k_{\text {- }}$ are intercrystalline cracks of equal depth producing a vertex split with width $\delta$ at the apex, rate constants of respectively forward and backward reactions. $\tilde{A}$ is a dimensionless specific interfacial surface per unit area, as above, per unit mass of pore fluid, $M_{f}{ }^{0}$ at which the reaction occurs. The applicability of the above reaction rate expression to in situ conditions is a separate, widely debated issue (see e.g. Steefel and MacQuarrie [22]), which will be addressed later on.

In what follows we will use the rate of change of activity of silicic acid in pore water to calculate the rate of relative mass removal of silica from the solid

$$
\dot{\xi}=s \frac{d a_{\mathrm{H}_{4} \mathrm{SiO}_{4}}}{d t}
$$

where $s$ is a molar ratio between water and silica in the initial volume of the material. The number of moles of silicic acid produced is equal to that of silica removed from the solid. Having normalized the above rate using the ratio $s, \xi$ is constrained by the inequalities: $0 \leq \xi \leq 1$. When $\xi=1$, the reaction is completed, that is all silica is removed from the material.

A new approach adopted in this paper consists in taking account of an increased dissolution occurring at the sites at the newly generated internal surface area of solid/fluid interface formed by the walls of microcracks. A hidden scalar variable is introduced representing the amount of the added surface area, $\tilde{a}$, per unit volume of the porous medium. The total surface area of microcracks can be measured directly in experiments and is proportional to the total acoustic energy released during cracking and measured by acoustic emission. Variable $\tilde{a}$ needs to be linked to the relative reaction area, $\tilde{A}$, and hence related to the volume of water with which the solid is reacting. Therefore an appropriate conversion is introduced 


$$
\tilde{a}=\tilde{A} \frac{n \rho_{w}}{\rho^{0}}
$$

where $\rho^{0}=1 \mathrm{~kg} / \mathrm{m}^{3}, \rho_{w}$ is the density of water, $n$ is porosity. The cumulative frequency of acoustic emission events is known as proportional to the inelastic dilatant volumetric strain, Brace et al. [7], Scholz [25]. Using a regular, 2D, hexagonal crystal assembly with two the ratio of the new internal interface surface area generated by cracking per unit volume of the medium, to the volumetric strain, $\varepsilon_{v}^{i r r}$, is $\tilde{a} / \varepsilon_{v}^{i r r}=8 /(\sqrt{3} \delta)$. If the increase in volumetric strain is attributed to the increase in the number of the cracks only, and not to their width, the ratio remains constant.

Using the above two assumptions it seems reasonable that the rate of increase generated by cracking of new surface area of the internal fluid/solid interface per unit volume of the medium be related linearly to the rate of inelastic volumetric strain (dilatancy)

$$
\tilde{a}=\chi^{\prime} \varepsilon_{v}^{i r r}+\tilde{a}_{0}, \varepsilon_{v}^{i r r}<0
$$

where $\chi^{\prime}$ is a coefficient of proportionality between the dilative volumetric strain and the relative surface area increase, taking also into account that not the whole area generated by dilatancy contributes to dissolution of quartz, which may constitute only a fraction of the rock. In addition we impose that $\chi^{\prime}=0$, if $\varepsilon_{v}^{i r r}>0$, that means when the irreversible volumetric strain is compactive it does not generate any cracks. $\widetilde{a}_{0}$ is the initial pre-existing pore interface area per unit medium volume, which produces a basal dissolution. Hence, for $\varepsilon_{v}^{i r r}>0$, $\tilde{a}=\tilde{a}_{0}$.

The reaction rate, eq. (8) has been obtained in laboratory conditions (Rimstidt and Barnes, [21]), where quartz grains were exposed to water for a period of time. In the context of microfracturing rocks several modifications need to be introduced to it. First, the surface areas considered in (8) must be re-scaled to be related to the representative elementary volume $(R E V)$ of the porous medium, as in (10). Second, it needs to be realized that within a $\mathrm{REV}$, the dissolution sites and precipitation sites do not necessarily coincide and not even have to be located within the same microcrack. A short-distance diffusive transport on the scale of the characteristic dimension of the REV may be involved, with dissolution being a controlling step, in most cases, as suggested by Berner [26]. However, applying eq. (8) to the REV scale allows us to include this transport in the kinetic rate.

Third, determination of the reaction area is considered as a factor of recurring difficulty (Lichtner [27]), especilly when the volume fraction of quatrz is variable due to dissolution. Lichtner suggests that the current reaction area is proportional to the current mineral volume fraction to the power of $2 / 3$. In the proposed model the variation of the dissolution site area is connected to micro-fracturation occurring during the damage process, through eq. (11). As for precipitation, it may occur either locally, at remote sites, or may not be occurring at all (Steefel and MacQuarrie [22]). Walderhaug ([28, 29]) suggests that the reaction area of precipitation of quartz during cementation of sandstone, is substantially reduced due to several processes, mainly a progressive grain and/or pore-wall coating, and other forms 
of contributions to porosity reduction. In what follows the dissolution reaction area $\tilde{a}_{d}$ isdifferentiated from that of precipitation $\tilde{a}_{p}$, so that

$$
\dot{\xi}=s \frac{d a_{\mathrm{H}_{4} \mathrm{SiO}_{4}}}{d t}=s \gamma_{\mathrm{H}_{4} \mathrm{SiO}_{4}}\left(\tilde{A}_{d} k_{+} a_{\mathrm{SiO}_{2}} a_{\mathrm{H}_{2} \mathrm{O}}^{2}-\tilde{A}_{p} k_{-} a_{\mathrm{H}_{4} \mathrm{SiO}_{4}}\right) ; \quad \widetilde{A}_{i}=\widetilde{a}_{i} \frac{\rho^{0}}{n \rho_{w}} ; i=p, d
$$

Here, the dissolution reaction area $\tilde{A}_{d}$ is linked directly to dilatancy trough eq. (11). For precipitation, the availability of this surface area is reduced progressively as a direct function of time, shown in Fig. 2, following the proposal of Walderhaug ([28])

$$
\tilde{a}_{p}=\tilde{a}_{p 0}^{\prime} \exp \left[-\left(\frac{t}{t_{c}}\right)^{2}\right]
$$

where $t_{c}$ is a constant defining the time of complete disapearance of the surface area available for precipitation, and $\tilde{a}_{p 0}^{\prime}$ is the initial specific area available for precipitation. Hence, the reaction rate (eq. 9) applied to the considered situation yields the following rate of relative mass removal

$$
\begin{aligned}
& \dot{\xi}=s \frac{d a_{\mathrm{H}_{4} \mathrm{SiO}_{4}}}{d t}=s \gamma_{\mathrm{H}_{4} \mathrm{SiO}_{4}}\left\{\left(\chi \varepsilon_{v}^{i r r}+\tilde{a}_{0}\right) k_{+} a_{\mathrm{SiO}_{2}} a_{\mathrm{H}_{2} \mathrm{O}}^{2}-\tilde{a}_{p 0} k_{-} a_{\mathrm{H}_{4} \mathrm{SiO}_{4}} \exp \left[-\left(t / t_{c}\right)^{2}\right]\right\} \\
& \chi=\chi^{\prime} \frac{\rho^{0}}{n \rho_{w}}, \tilde{a}_{p 0}=\tilde{a}_{p 0}^{\prime} \frac{\rho^{0}}{n \rho_{w}}
\end{aligned}
$$

As a result it appears that the chemical softening variable rate is explicitly a function of time, the accumulated irreversible strain, and of the activity of silicic acid. Integration of eq. (14) must be coupled to that of eqs. (3) and (6).

In what follows, a closed system (a representative elementary volume) in which dissolution is partially counterbalanced by a local precipitation is presented. An open system, a boundary value problem in fact, which is affected by the local dissolution and a long-range diffusion away from a dissolution site, while the local precipitation is considered negligible is discussed elsewhere $\mathrm{Hu}$ and Hueckel [31].

\section{CREEP AS A RESULT OF LOCAL DISSOLUTION AND PRECIPITATION}

Consider a process of chemical weakening of a material affected by dissolution and precipitation processes which are both local. In such case the product of dissolution is subsequently precipitated within the same representative elementary volume on the interface surface area available. The net amount of the species that is removed from the solid is a result of different rates with which dissolution and precipitation develolp. That does not imply that precipitation occurs at the wall surface of the same microcrack where dissolution take places. It does however entail that transport of dissolved species, if any, is limited to the interior of the representative elementary volume. Such possible transport and other phenomena eventually affecting the chemical equilibrium are also discussed together with possible causes of creep failure mechanisms.

Phenomenologically, three phases of creep strain development with time are usually identified. They are referred to as primary, secondary and tertiary phase (Fig. 3), all preceded 
by a phase of an elastic strain. The latter phase is not present in this analysis as the material is considered as rigid-plastic. Still, some strain due to stress state required to be reached within the plastic domain may be present prior to the creep stage. Primary creep is characterized by an initial period with a strain rate which is relatively high, but in a well marked transition period deccelerates to a constant rate. Secondary creep is characterized by constant rate of strain. Tertiary creep indicates a phase of a rapidly accelerating strain, ending possibly with a very high, possibly infinite, strain rate and hence failure.

The mechanisms which in this model activate the above described creep phases are: dissolution and precipitation, that together with the corresponding (variable) reaction surface areas affect the variable of mass change of silica, and finally two hardening function: strain hardening and chemical softening, which control the change in the material strength. Those mechanisms are discussed in detail in what follows.

The material strength is defined by yield limit in terms of stress. A specific yield limit adopted here is an adaptation of a parabolic yield function developed by Dragon and Mróz, [30],

$$
f\left(\sigma_{i j}, \kappa, \xi\right)=s_{i j} s_{i j}-2 p_{h} \cdot\left(I_{1}^{0}-\sigma_{k k}\right)
$$

where $s_{i j}$ is deviatoric stress, while $p_{h}$ is hardening parameter and $I_{l}^{0} /\left(2 p_{h}\right)$ is an isotropic stress value, at which the yield limit intersects the isotropic stress axis.

The current size of the yield locus, $p_{h}$ is a resultant of a mechanical and chemical hardening taken respectively as a linear deviatorc strain hardening and a logarithmic chemical softening, that is

$$
p_{h}(\kappa, \xi)=p_{0}\left[1+\alpha_{0} \kappa-\beta_{0} \ln \frac{\beta_{1}-\xi_{0}}{\beta_{1}-\xi}\right]
$$

in which the reaction progress $\xi$ is expressed through its rate in terms of aqueous silica activity rate, via eq. (15). $\alpha_{0}, \beta_{0}$ are material constants. $\beta_{1}$ is a constant defining the chemical softening curve as in Fig. 3, which describes the chemical softening as explained below. The material remains in full strength when no chemical softening occurs, while looses all its strength when completely depleted of silica. The loss of strength is limited at the beginning of mass depletion, and at $75 \%$ of depletion is still less than $30 \%$ of the initial strength. However, beyond that range the loss of strength is far more dramatic.

Parameter $p_{h}=p_{0}$ at no hardening is related to the pre-peak yielding stress value, $k f_{\mathrm{c}}$, during uniaxial compression of the material, with $0.4 \leq k \leq 0.6, f_{c}$ is the uniaxial strength, via (Dragon and Mróz [30])

$$
p_{0}=1 / 3 \frac{k^{2} f_{c}^{2}}{I_{1}^{0}-k f_{c}}
$$

In what follows the initial dissolution specific surface $\tilde{a}_{0}$ in eq. (15) is assumed to be zero, all the interface surface being created by dilatancy.

At constant stress and hence $p_{h}=$ const, $s_{k l}=$ const, the volumetric strain (dilatancy) can then be determined by solving the following system of differential equations obtaining $\varepsilon_{v}$ and $\xi$, asa function of time $t$, assuming that at $t=0, a_{H_{4} S_{i O}}=0$ :

$$
\dot{\varepsilon}_{v}=\frac{\beta_{0}}{\left(\beta_{1}-\xi\right) \alpha_{0}} \frac{3 p_{h}}{\left(\frac{2}{3} s_{k l} s_{k l}\right)^{1 / 2}} \dot{\xi}
$$




$$
\dot{\xi}=s \chi \varepsilon_{v} \gamma_{\mathrm{H}_{4} \mathrm{SiO}_{4}} k_{+} a_{\mathrm{SiO}_{2}} a_{\mathrm{H}_{2} \mathrm{O}}^{2}-\tilde{a}_{p 0} k_{-} \xi \exp \left[-\left(t / t_{c}\right)^{2}\right]
$$

Eqs. (18) and (19) define the creep behavior of the material in question.

Substituting the chosen constitutive functions into eq. (6) one arrives at creep strain rate expressed by the reaction rate as follows

$$
\dot{\varepsilon}_{i j}=\frac{\partial \beta / \partial \xi}{\partial \alpha / \partial \kappa} \frac{s_{i j}+p \delta_{i j}}{\left(2 / 3 s_{k l} s_{k l}\right)^{1 / 2}} \dot{\xi}
$$

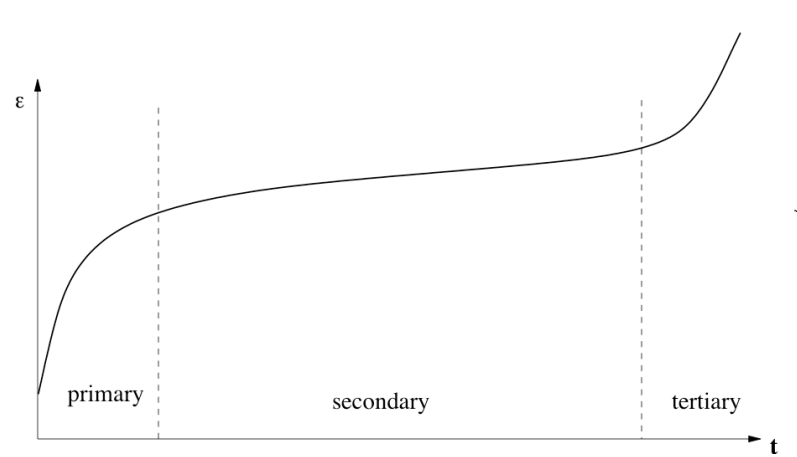

Figure 3. A typical three-phase creep curve

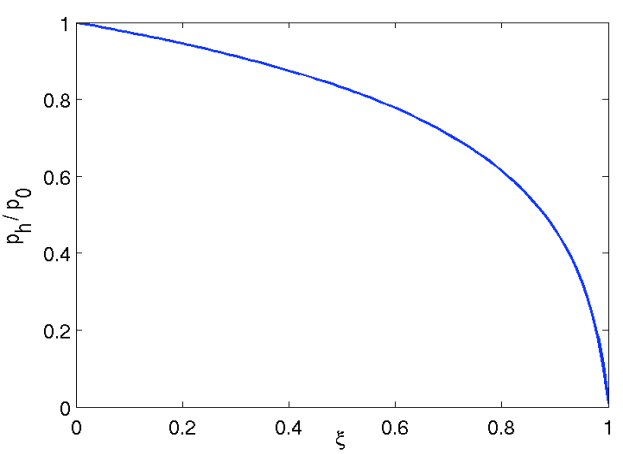

Figure 4. Logarithmic chemical softening function

The hardening function in eq. (17) as a linear deviatorc strain hardening and a logarithmic chemical softening, that is

\section{NUMERICAL SIMULATIONS}

Consider a biaxial compression of a homogeneous isotropic material element loaded symmetrically with the stress components $\sigma_{2}=\sigma_{3}, \sigma_{1}=0$. The integration of the system (18, 19) has been performed numerically using Mathlab 7.0. Once the equation system is solved, the creep strain rate components can be obtained as:

$$
\begin{aligned}
& \dot{\varepsilon}_{1}=\frac{\beta_{0}}{\left(\beta_{1}-\xi\right) \alpha_{0}} \frac{s_{11}+p_{h}}{\left(\frac{2}{3} s_{k l} s_{k l}\right)^{1 / 2}} \dot{\xi} \\
& \dot{\varepsilon}_{2}=\dot{\varepsilon}_{3}=\frac{\beta_{0}}{\left(\beta_{1}-\xi\right) \alpha_{0}} \frac{s_{22}+p_{h}}{\left(\frac{2}{3} s_{k l} s_{k l}\right)^{1 / 2}} \dot{\xi}
\end{aligned}
$$

The activity coefficients of silica and water are assumed to be unity. The constitutive parameters and rate constants are chosen as shown in Table 1: 
Table 1

\begin{tabular}{|c|c|c|c|c|c|c|c|c|c|c|c|}
\hline $\mathrm{I}_{1}{ }^{0}$ & $\mathrm{p}_{0}$ & $\mathrm{~s}$ & $\chi$ & $\tilde{a}_{p 0}$ & $\alpha_{0}$ & $\beta_{0}$ & $\beta_{1}$ & $\xi_{0}$ & $\mathrm{k}_{+}$ & $\mathrm{k}_{-}$ & $\mathrm{K}$ \\
$\mathrm{MPa}$ & $\mathrm{MPa}$ & & & & & & & & $\mathrm{sec}^{-1}$ & $\mathrm{sec}^{-1}$ & \\
\hline 63.04 & 0.095 & 3.33 & $1 \times 10^{6}$ & $0.12 \times 10^{6}$ & 1 & 0.252 & 1.02 & 0 & $6.09 \mathrm{E}-11$ & $3.76 \mathrm{E}-10$ & 0.16 \\
\hline
\end{tabular}

The dissolution rate constant has been taken directly as that of Rimstidt and Barnes, [20]. The rate constant for precipitation is discussed in a parametric study, which follows. First, creep is analyzed at different stress levels with the rate constants provided above. Creep strain is simulated for three particular stress states: i) $\sigma_{1}=0, \sigma_{2}=\sigma_{3}=-4.832 \mathrm{MPa}$; ii) $\sigma_{1}=0$, $\sigma_{2}=\sigma_{3}=-6.569 \mathrm{MPa}$; iii) $\sigma_{1}=0, \sigma_{2}=\sigma_{3}=-7.691 \mathrm{MPa}$. The corresponding initial strain components at the onset of creep are calculated for an instantaneous rigid-plastic behavior: i) $\varepsilon_{1}^{0}=0.022, \varepsilon_{2}^{0}=\varepsilon_{3}^{0}=-0.010$; ii) $\varepsilon_{1}^{0}=0.116, \varepsilon_{2}^{0}=\varepsilon_{3}^{0}=-0.051$; iii) $\varepsilon_{1}^{0}=0.190, \varepsilon_{2}^{0}=\varepsilon_{3}^{0}=-0.082$.

The resulting deviatoric creep strain and its strain rate are shown in Fig. 5a and $5 \mathrm{~b}$ as a function of time for the three stress levels. Significantly larger strain devolops at higher stress levels. The transitions between the primary stage, secondary stage and tertiary stage also occur quicker at a higher stress because of a larger dilatancy and hence, an accelerated dissolution. The character of creep curves is well reproduced by the mechanisms postulated.
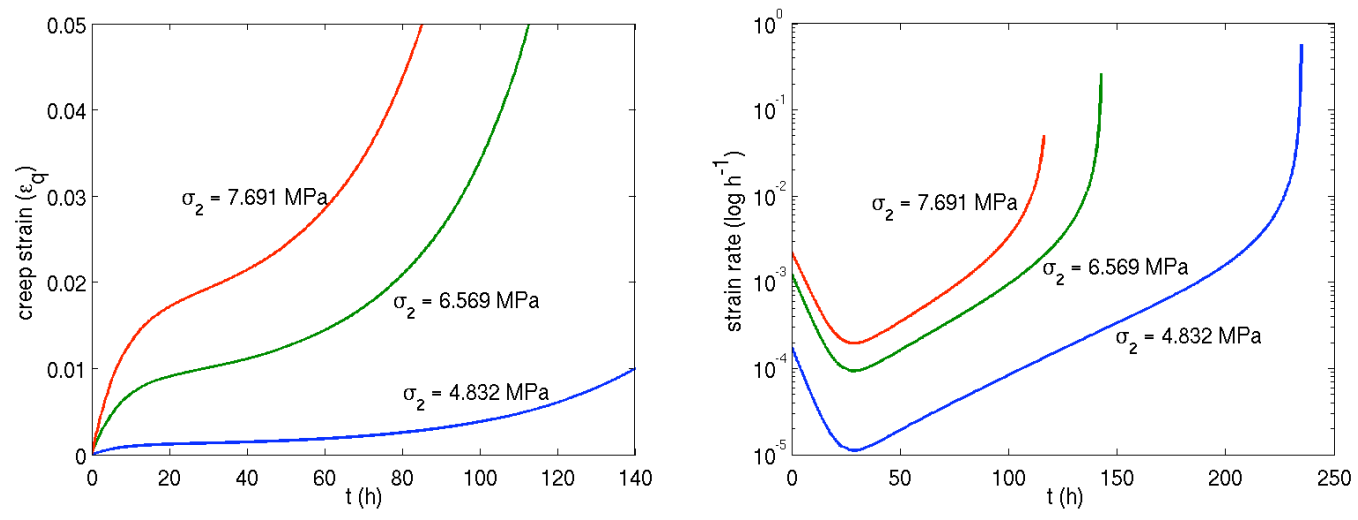

Figure 5. Deviatoric creep at different stress levels: (a) strain, and (b) strain rate

Our aim now is to see how the three phases of creep relate to the variable contributions of the mechanisms involved: the mechanical one of strain hardening, controlled by deviatoric strain $\varepsilon_{q}$ and the chemical mechanism controlled by the relative mass removal $\xi$. By virtue of the compensatory constraint triggered by the creep conditions, as mentioned earlier, the two variables become inter-dependent via eq. (18-19). Due to the logarithmic form of the chemical softening function, deviatoric strain is visibly augmented near the completion of mass removal. However, it has to be reminded that the theory becomes less accurate when we leave the small strain range. It is easy to see that integrating and subtracting eq. (21) a and $b$ 
yields a stress independent function, depicted in Fig. 6. This is not the case of the volumetric (dilative) strain, which generally increases with the value of the imposed stress, Fig. 7. The relative mass removal, $\xi$, is clearly enhanced by the acting compressive stress, Fig. 8a.

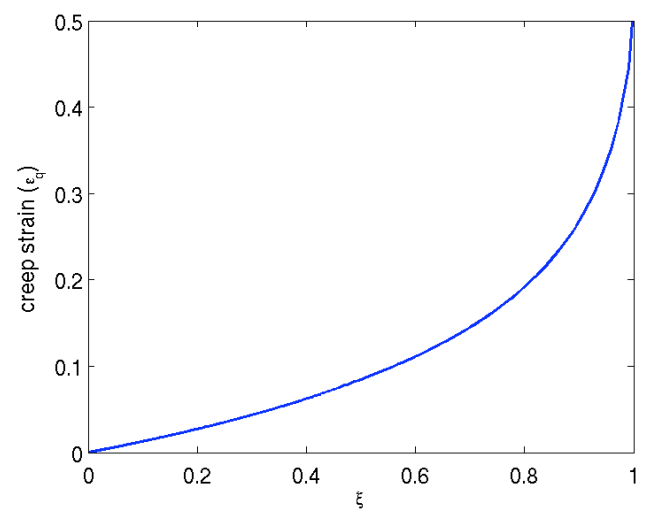

Figure 6. Deviatoric creep strain vs. $\xi$

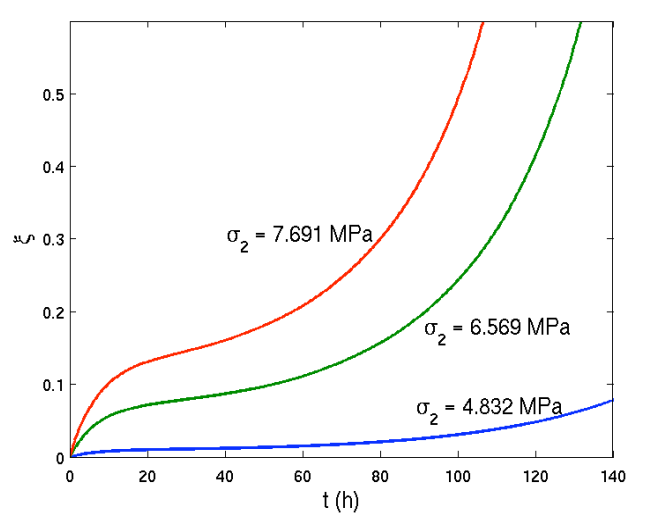

Figure 8a. Evolution of $\xi$

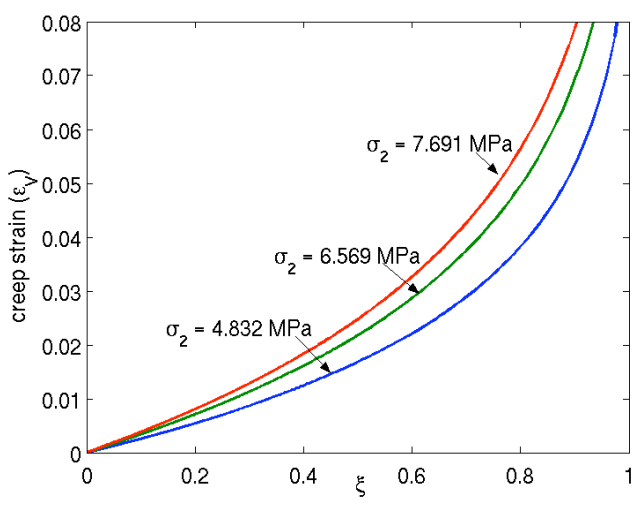

Figure 7. Volumetric creep strain vs. $\xi$

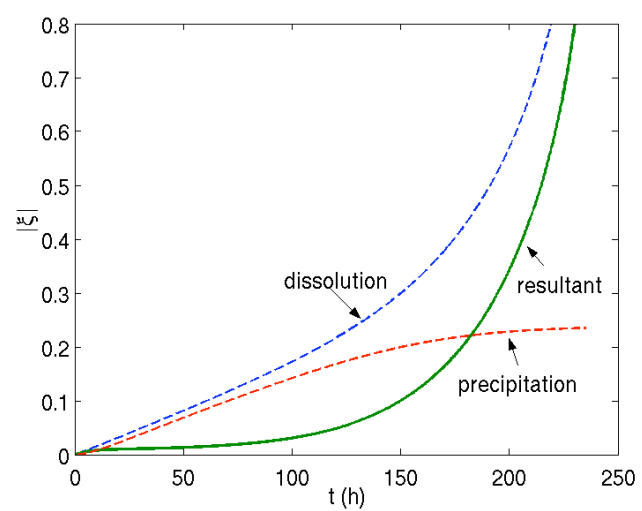

Figure $8 \mathrm{~b}$ : The components of $\xi$ at $4.832 \mathrm{MPa}$

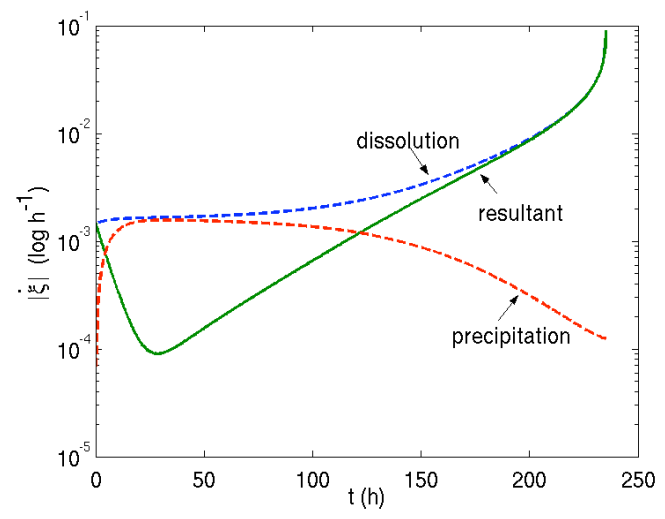

Figure $8 \mathrm{c}$. The components of the rate of relative mass removal at $4.832 \mathrm{MPa}$

The resultant relative mass removal, $\xi$, is affected by four factors: dissolution rate constant, the specific surface area available for this reaction, the constant of the rate of precipitation (which is subtracted) and the corresponding specific surface area available for this reaction (eqs. 11 and 13). The first stage of the relative mass removal, $\xi$, for less than 10 first 
hours shows the prevailing effect of dissolution part of the reaction progress, Fig. $8 \mathrm{a}$ and $8 \mathrm{~b}$. This is despite the fact that dissolution rate constant is about seven times smaller than that precipitation. However, the initial surface area which is also an important factor in the resultant specific reaction rate (eq. 11) is much higher for dissolution than that for precipitation. In fact, the initial surface area of dissolution depends directly on the dilatant volumetric strain accumulated during pre-creep loading stage, which increases with the level of stress, see Fig. 7. Additionally, for the physical reasons, the surface area for dissolution area increases as a result of damage/dilatancy, while that for precipitation it decreases due to accumulative grain and/or pore-wall coating. The above evolutions yield the partial and total rates presented in Fig. 8c.

It is hence concluded that primary creep is initially dominated by dissolution. As a result of an increasing precipitation, which reduces the net mass removal rate, the strain rate has a decelerating trend. Both the initial reaction rate and deviatoric strain rate, as well as the threshold deviatoric strain opening the secondary creep phase, are directly related to the stress level dependent, pre-creep dilatancy, that is also the micro-crack induced surface area. The latter feature represents the transition to the secondary phase in which while the creep strain increases, the strain rate is diminishing. This phase includes the mechanism of the reduction of the surface area of precipitation [(eq. 14), Fig. 1], as well as a monotonic increase of the activity of silicic acid $a_{\mathrm{H}_{4} \mathrm{SiO}_{4}}$. As the former one gradually starts to dominate over the latter effect, the overall mass removal rate and deviatoric strain rate begin to increase again relatively slowly. Notably, the minimum strain rate depends on the pre-creep damage, and through it on the stress level, Fig. 5b. Eventually, the tertiary phase is induced by the terminal phase of the chemical softening effect, for $\xi>0.75$. At this point the process is significantly accelerated toward the completion of the depletion of the mass, as described by the logarithmic chemical softening function $\beta(\xi)$, eqs. (16). Hence, it is consistent with the concept of failure nature of tertiary creep, which in this formulation is linked to chemical softening failure caused by mass removal.

It is interesting to note that the aforementioned compensatory mechanism regarding the hardening variables of eq. (4/) can be used to address the dual phenomenon of creep, which is relaxation. For simplicity, one may consider relaxation under a constrained deviatoric strain invariant, i.e. for $\dot{\kappa}=0$. The condition for the progress of the process of the chemo-plastic softening, eq. (4), under those conditions implies that $\left(\partial f / \partial \sigma_{i j}\right) \dot{\sigma}_{i j}<0$, for the relative mass removal $\dot{\xi}>0$, given the fact that for chemical softening $\partial f / \partial \xi>0$. Hence, the stress rate is required to be oriented into interior of the current yield locus, which in a 1-D case means that the stress is decreasing. However, to model properly the evolution of the reactive surface area via dilatancy may require considering an elastic strains or a strain or displacement component based relaxation condition.

\section{CONCLUSIONS}

A damage enhanced reactive chemo-plasticity model is developed to reproduce the creep behavior of saturated geomaterials. A closed system in which the chemical softening is affected by both local dissolution and precipitation is considered. An open system when a mass removal due to dissolution and long-range diffusion while local precipitation is neglected is considered elsewhere. Deformation at the constant load in the closed system exhibits all the classical creep charactersitics. The strain and strain rate are visibly higher under higher constant loads. The results show the central role of the compensatory mechanism between strain 
hardening and chemical softening. Primary, secondary and tertiary creep can be interpreted in terms of the role of the rates of dissolution and precipitation, as well as chemical softening intensity.

It may be concluded that the developed constitutive chemo-plasticity equations render the plasticity law explicitly rate dependent. The advantage of that dependency is its direct link to more fundamental concepts and laws of basic geochemistry. In addition the evolution of the material behavior through the individual phases of creep can be attributed to specific chemical and deformational mechanisms developing during the process. The above demonstration obviously does not free the potential user of the verification of the applicability of the specific constitutive assumptions and formulae to the geomaterial under consideration, as other sources of micro-scale rate dependence are possible.

The results demonstrate a validity of the hypothesis that chemo-mechanical damage process, enhanced spontaneously in time by a basic geochemical reaction, produces deformation that has all the characteristics of creep. An application of the discussed theory to specific microscale processes of intergranular contact penetration is discussed elsewhere $(\mathrm{Hu}$ and Hueckel [31]). Finally, it needs to be reminded that chemical reactions are not the only possible cause of creep. Other phenomena, such as transfer of water from or to micropores that are explicitly time dependent may also contribute to rate dependence of plasticity, and hence creep, see e.g. Murad et al.,[17], Navarro and Alonso [32].

\section{ACKNOWLEDGMENT}

This work was partially supported by the US National Science Foundation, grant \# 0324543, Geomechanics and Geotechnical Systems Program.

\section{REFERENCES}

[1] M. E. Kassner, and Perez-Prado, M-T., Fundamentals of creep in metals and alloys, Elsevier, Amsterdam, pp. 272, 2004

[2] S. Murayama, Shibata T. Rhelogical proerties of clays. Proceedings of the fifth International Conference on Soil Mechanics and Foundation Engineering; 1:269-273, 1961

[3] R.W. Christensen, Wu PL. Analysis of clay deformation as the rate process. Journal of the Soil Mechanics and Foundations Division. ASCE 1965; 91(SM5):125-157

[4] J.K. Mitchell Shearing resistence of soils as a rate process. Journal of the Soil Mechanics and Foundations Division, ASCE; 90(SM1):29-61, 1964

[5] M.R. Kuhn, Mitchell JK. New perspectives of on soil creep. Journal of Geotechnical Engineering, ASCE; 119(3):507-524, 1993

[6] W.F. Brace, Paulding BW, Scholz, CH. Dilatancy in the fracture of crystalline rocks. Journal of Geophysical Research 1966; 71:3939-3953 
[7] A.J., Gratz, Solution-Transfer Compaction of Quartzites - Progress Toward a Rate Law, Geology, 19 (9): 901-904, 1991

[8] R. Tada, Siever R. Experimental knife-edge pressure solution of halite. Geochim. Cosmochim. Acta; 50:29-36, 1986

[9] K.L. Milliken,. The widespread occurrence of healed microfractures in siliciclastic rocks: Evidence from scanned cathodolumescene imaging. PP Nelson and SE Laubach eds, Proceedings of the North American Rock Mechanics Symposium, Austin, TX. Balkema, Rotterdam, $825-832,1994$

[10] S.W.J. den Brok. Effect of microcracking on pressure solution strain rate: The Gratz grain-boundary model. Geology, 26(10):915-918,1998;

[11] P.M.T.M. Schutjens, Spiers CJ. Intergranular pressure solution in NaCl: grain-to-grain contact experiments under the optical microscope. Oil \& Gas Science and Technology-Rev. IFP; 54(6): 729-750, 1999

[12] W. W. He, A. Hajash and D. Sparks, Creep compaction of quartz aggregates: effects of pore-fluid flow- a combined experimental and theoretical study, Americal Journal of Science, 303, 73-93, Feb., 2003

[13] B.K. Atkinson. Subcritical crack growth in geological materials. Journal of Geophysical Research; 89: 4077-4114, 1984

[14] T. Hueckel, Water-mineral interaction in hygro-mechanics of clays exposed to environmental loads: a mixture theory approach. Canadian Geotech. Journal; 29:1071-1086, 1992

[15] T. Hueckel. Reactive plasticity for clays during dehydration and rehydration. Part I: concepts and options. International Journal of Plasticity 2002; 18(3):281- 312

[16] T. Hueckel. Chemo-plasticity of clays subjected to flow of a single contaminant and stress. International Journal for Numerical and Analytical Methods in Geomechanics 1997; 21(1):43-72

[17] M. Murad, J. Guerreiro, A. F.D. Loula, Micromechanical computational modeling of secondary consolidation and hereditary creep in soils, Comput. Methods Appl. Mech. Engrg. 190 $1985 \pm 2016,2001$

[18]Hueckel T, Borsetto M. Thermoplasticity of saturated soils and shales: constitutive equations. Journal of Geotechnical Engineering, ASCE 1990; 116(12):765-1777

[19]. Liu Z, Boukpeti N, Li X, Collin F, Radu JP, Hueckel T, Charlier R. Modelling chemohydro-mechanical behaviour of unsaturated clays: a feasibility study. International Journal for Numerical and Analytical Methods in Geomechanics 2005; 29:919-940

[20]. Rimistid JD, Barnes DL. The kinetics of silica-water reaction, Geochim. Cosmochim. Acta 1980; 44:1683-1699 
[22] Steefel CI, MacQuarrie KTB. Approaches to modeling of reactive transport in porous media. Reactive Transport in Porous Media. PC Lichtner, CI Steefel and EH Oelkers, eds. Mineralogical Society of America; 1996

[25] Scholz C. Micro fracturing and the inelastic deformation of rock in compression. Journal of Geophysical Research; 73:1447-1454,1968

[26] Berner RA. Rate control of mineral dissolution under earth surface conditions. American Journal of Science.; 278:1235-1252,1978

[27] Lichtner PC. Continuum formulation of multicomponent-multiphase reactive transport. Reactive Transport in Porous Media. P.C.Lichtner, CI Steefel and EH Oelkers, eds. Mineralogical Society of America, 1996

[28] O. Walderhaug Kinetic modeling of quartz cementation and porosity loss in deeply buried sandstone reservoirs. The American Association of Petroleum Geologists Buttletin 80(5):731-745,1996;

[29] O. Walderhaug Modeling quartz cementation and porosity in middle Jurassic Brent group sandstones of the Kvitebjorn field, Northern North Sea. The American Association of Petroleum Geologists Buttletin; 84(9):1325-1339, 2000

[30] A. Dragon, Mróz Z. A model for plastic creep of rock-like materials accounting for the kinetics of fracture. International Journal of Rock Mechanics and Mining Sciences 16:253259,1979;

[31] Hu LB, Hueckel T. Coupled chemo-mechanics of intergranular contacts. Computers and Geotechnics, to appear, 2006

[32] V. Navarro, Alonso E.E. Secondary compression of clays as a local dehydration process Geotechnique, 51 (10): 859-869,2001 